\title{
Synthesis and Structural Characterization of Sulfur Nanowires.
}

\author{
P.Santiago, * E. Carvajal,** D. Mendoza, ***, and L. Rendón* \\ * Instituto de Física, Universidad Nacional Autonoma de México, A.P. 20-364, 01000 México \\ ** Facultad de Ciencias, U.N.A.M., A. P. 70-646, 04510 México, D.F. \\ *** Instituto de Investigaciones en Materiales, U.N.A.M., A.P. 70-360, 04510 México, D.F.
}

Sulfur is one of the most studied elements due to its technological applications. For example, as molecular electronic devices, sulfur has the susceptibility to bond with gold and other metal surfaces [1]. It is also well known that sulfur plays an important role in the chemistry of interstellar medium and used to form stable carbon chains such as $\mathrm{C}_{3} \mathrm{~S}$ and $\mathrm{C}_{5} \mathrm{~S}$, actually carbon nanotubes have been functionalized with these $\mathrm{C}-\mathrm{S}$ chains in order to obtain hybrid materials with useful properties for gas sensor and catalytic applications [2].

In the present work we report the synthesis of single crystalline Sulfur nanowires at room temperature using anodic alumina nanoporous templates (AANTs) with an average porous innerdiameter of $20 \mathrm{~nm}$ [3]. We used S-CS $2(10 \mathrm{~g}$ in $40 \mathrm{ml})$ as solution phase precursor. The AANT was immersed in the precursor solution for 5 days in a special flask to fill the alumina porous. The AANT's were dried at room temperature in order to evaporate the $\mathrm{CS}_{2}$ remaining solvent in a safe extractor system. The structural characterization was performed in a JEM 2010 FasTem microscope equipped with a Noran EDS spectrometer. The sample was prepared for its observation using a mortar in order to crack the alumina mold and liberates the nanowires without any further chemical process.

From the TEM studies we found both curly and straight nanowires. The sulfur nanowires diameter is between 15 to $20 \mathrm{~nm}$ and about $1000 \mathrm{~nm}$ length. Figure 1b) corresponds to a HRTEM micrograph where is possible to observe the lattice space parameter which corresponds to $d=0.295 \mathrm{~nm}$. This lattice parameter is in good agreement with the reflection (140) for the Monoclinic $\mathrm{S}_{8}$ phase (PCPDF file 86-1278), however the structural characterization is still under study. There are also bending wires (figure 2a) where it is possible to observe stacking faults defects. A chemical elemental microanalysis was also performed in nanobeam mode (figure 2b); the carbon peak observed corresponds to the grid support. In some monocristalline wire zones we could observe that the preferential grown axis corresponds to [140] for the monoclinic Sulphur structure, however we are studying the phase under mathematical simulated methods in order to understand the structure.

Differential scanning calorimetry measurements were also performed for this system. However, the preliminary results are not in agreement with the bulk sulfur data reported in the literature. We found that the characteristic temperatures associated with the bulk sulfur transformations are different to the corresponding with our 1-D sulfur structures.

[1] M.G. Samant, C.A. Brown, J.G. Gordon, Langmuir 8 (1992) 1615.

[2] R. Zanella, E. Basiuk, P. Santiago, V. A. Basiuk, E. Mireles, I. Puente-Lee, and J.M. Saniger., J. Phys. Chem. 109, (2005) 16290.

[3] C.Peza-San German, P. Santiago, et. al., J. Phys. Chem. B 109 (2005) 17488.

[4] We kindly acknowledge to Instituto de Física at UNAM for allowing the use of their microscopy facilities. This work is supported by a grand from CUDI-CONACyT. We also acknowledge the assistance of Mr. Angel Flores and Diego Quiterio in the sample preparation process. 


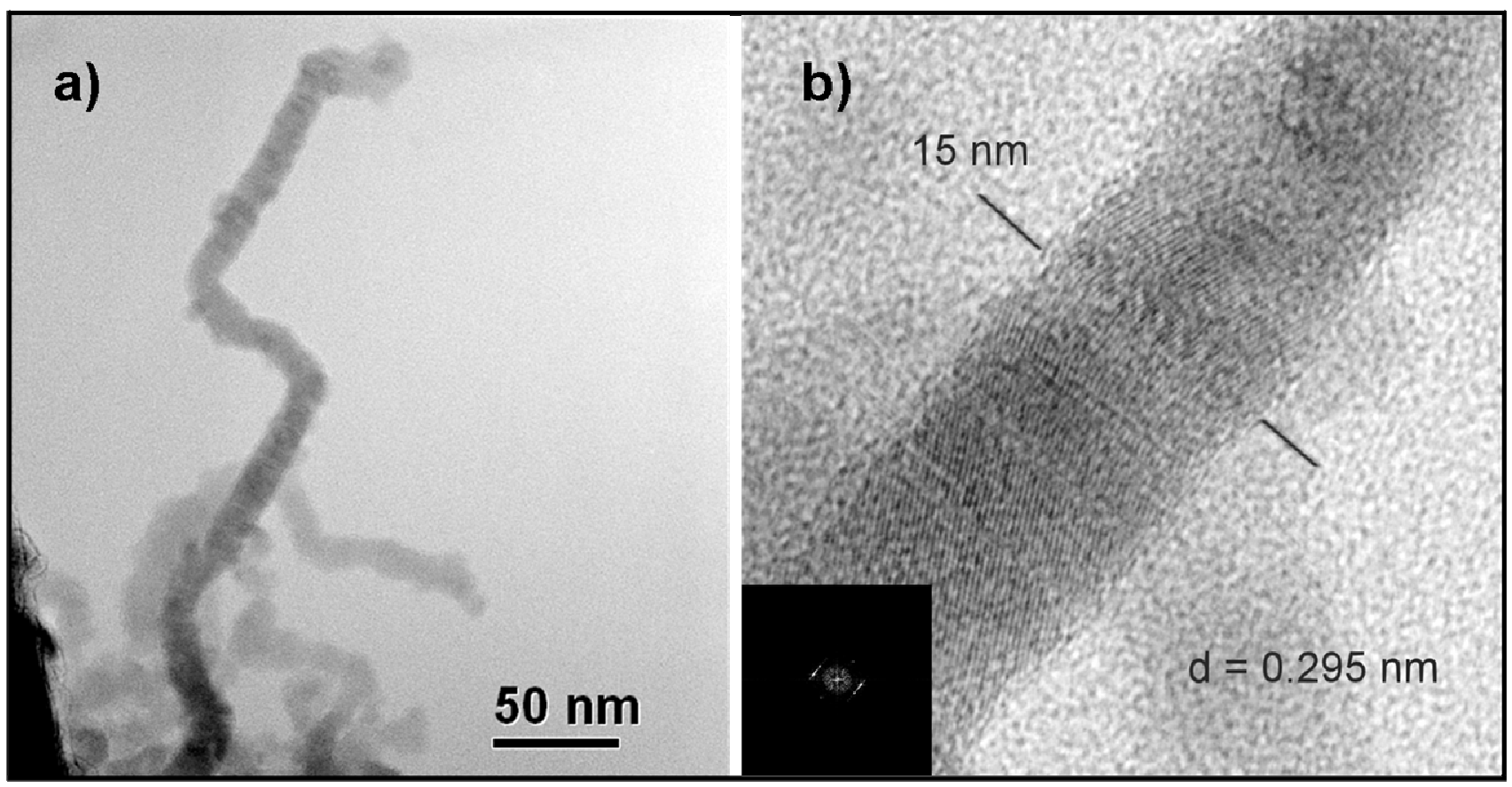

Figure 1. a) Conventional TEM micrograph of 1-D S structure. b) HRTEM of straight S NW..

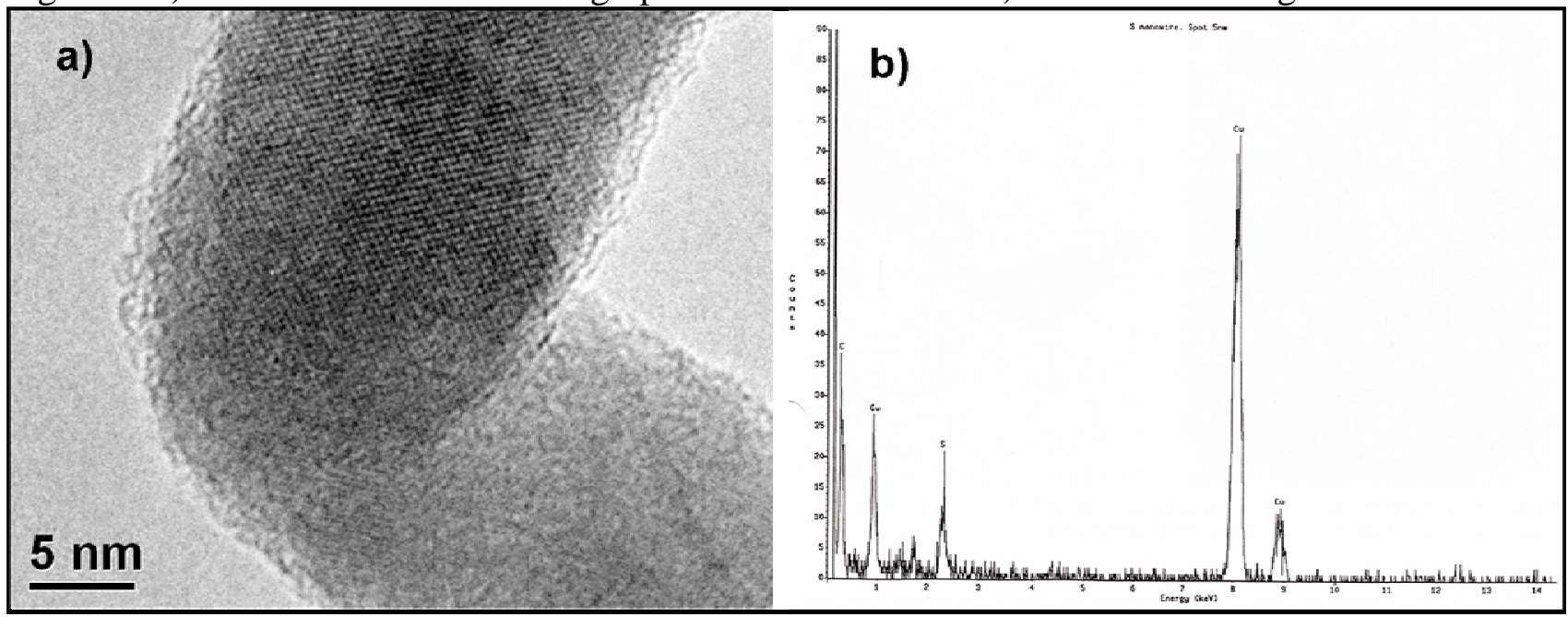

Figure 2. a) HRTEM micrograph of a curly S-NW. b) EDS microanalysis obtained in nanobeam mode (5 nm spot).

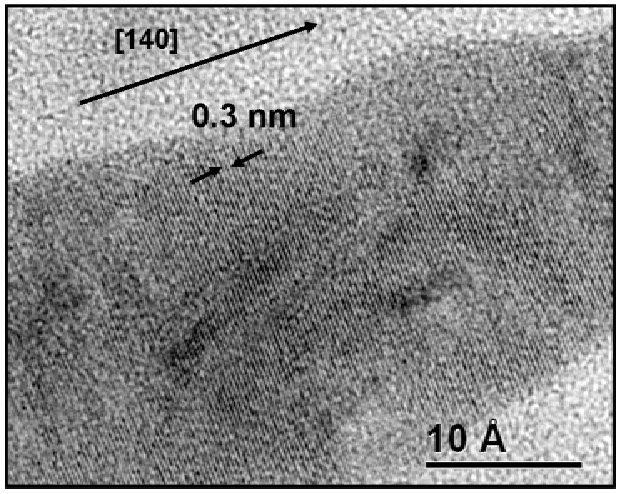

Figure 3. a) HRTEM micrograph of single S monoclinic nanowire growing in [140] direction. 